\title{
MODELING AND MEASUREMENTS OF THE DUVFEL PHOTOINJECTOR CAVITY RF PROPERTIES*
}

\author{
J. Rose, W. Graves, R. Heese, E. Johnson, S. Krinsky, B. Sheehy \\ NSLS, BNL, Upton, NY 11973, USA
}

\begin{abstract}
The 1.6 cell S-band photoinjector cavity has been in operation at the DUVFEL at BNL for the past year. Initial beam measurements indicated a higher than expected emittance and beam divergence at the exit of the gun. field imbalance in the two cells was suspected, but lack of field probes in the cavity prohibited direct confirmation. A detailed SUPERFISH model was constructed of the cavity assembly. Field balance as a function of cathode plate and tuner position was determined using the model. A series of calibrated measurements of both the cavity RF properties and the delivered RF power was undertaken. Electron beam properties were also measured as a function of cavity tune. Significant improvements in beam quality were achieved. The simulations and measurements are presented.
\end{abstract}

\section{INTRODUCTION}

The Deep UltraViolet Free Electron Laser (DUVFEL) project at BNL [1],[2],[3],[4] has a 210MeV electron linac for a driver. This linac consists of a 1.6 cell photocathode electron gun followed by a focusing solonoid, two SLAC type $2856 \mathrm{MHz}$ traveling wave accerating tanks which bring the energy to $77 \mathrm{MeV}$, a bunch compression chicane and two additional accelerating tanks which bring the final energy to 200 $\mathrm{MeV}$. This linac arrangement will produce sub-picosecond bunches of $3 \times 10^{9}$ electrons for injection into the NISUS undulator. The photocathode gun and first two linac tanks are powered by a single Thompson klystron tube (Type 2128A, 45MW) Another two similar klystrons power tanks three and four. During the course of commissioning the photocathode gun several discrepancies between theory and measurement of the RF performance of the gun and of the electron beam were noticed. A program to fully characterize the RF properties of the gun was undertaken and resulted in improvements to the cathode geometry.

\section{ORIGINAL CAVITY GEOMETRY}

The photocathode cavity [5] is a 1.6 cell cavity operated in the pi mode. A Superfish [6] plot is shown in figure 1.

The original cavity geometry used a copper Helicoflex Delta $^{\mathrm{TM}}$ vacuum seal in a groove in the cathode for both the ultra-high vacuum seal and to carry the rf currents. The seal is captive in a groove in the cathode which is recessed $\sim 5 \mathrm{~mm}$ from the cavity interior. The rf path length from the cavity interior, through the narrow gap to the groove, across the seal and back is $20 \mathrm{~mm}$. Since the rf wavelength at $2856 \mathrm{MHz}$ is $105 \mathrm{~mm}$, this path length is nearly a quarter wave. To make matters worse, the cathode is assembled in a vertical orientation, which causes the seal to rest on the top of the groove. This makes the path length shorter at the top and longer at the bottom. This was sufficient to cause arcing across the narrow gap between the cavity body and the cathode plate, (top left of figure 1). Extensive pitting of the cathode plate and cavity body in this gap was evident after several months of running. A redesign of the cathode plate to incorporate an RF spring seal was undertaken to remedy the problem.

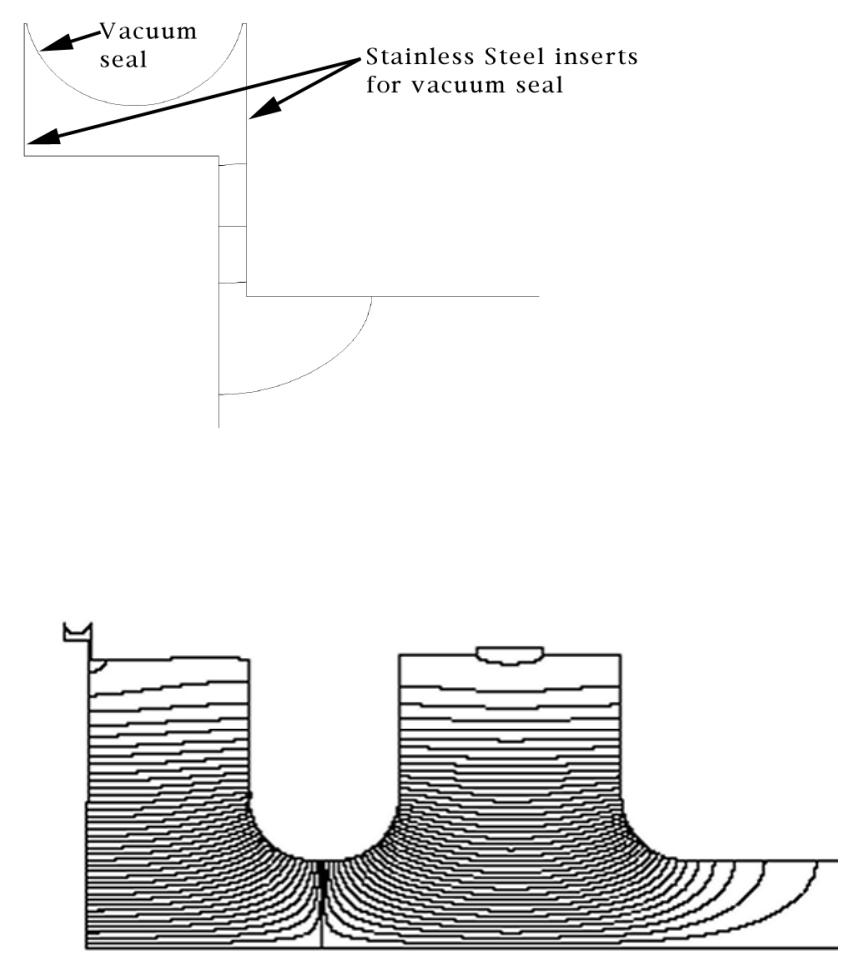

Figure 1. BNL/UCLA/SLAC Gun IV Geometry.

The SUPERFISH modeling of the old geometry found that the stainless steel inserts which form the bottom of the vacuum groove surface in the cathode plate and mating surface in the cavity body have a noticeable effect on the cavity Q. The losses in the stainless steel are over 6 times that in the copper. So although the RF currents pass over only a $5 \mathrm{~mm}$ annular ring of the stainless steel a significant fraction of $\mathrm{rf}$ power is dissipated and this degrades the Q by $20 \%$. 


\section{NEW CATHODE GEOMETRY}

The cathode plate was redesigned to include an RF spring ring. In order to be compatible with the vacuum bake-out temperatures of 200 degrees $\mathrm{C}$, and also to avoid poisoning of the cathode a 316 stainless steel Bal-Seal ${ }^{\mathrm{TM}}$ spring, gold plated to 4 skin depths was used.

Because the photocathode gun is installed in an operating system all modifications had to be made to the cathode plate, which is easily replaced. The available space for adding the RF seal between the existing vacuum seal groove and the cavity interior is only $2.5 \mathrm{~mm}$. This limited the space available for the spring ring groove to approximately half this. A Bal-Seal ${ }^{\mathrm{TM}}$ RF100 series spring was used. A detail of the seal as modeled in Superfish is shown in Figure 3. The seal consists of a .007 inch 316 stainless steel wire in a spring of coil height/width of $.051 / .056$ inches. This was gold plated with 0.00025 inches of gold.

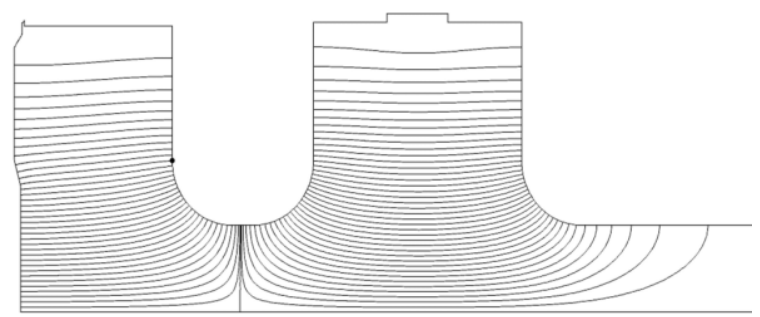

Figure 2: Cavity geometry with rf seal.

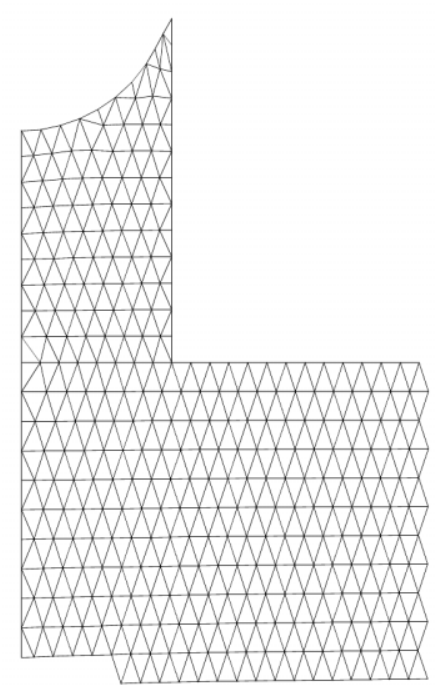

Figure 3. Detail of the Superfish model with RF seal (Upper left corner of Figure 2).

Superfish studies indicated that the cathode plate geometry would have to change to compensate for $20 \mathrm{MHz}$ frequency shift due to eliminating the volume of the re-entrant seal. The tuning effect of moving the entire cathode plate is nearly neutral since it is near the midplane of the $\mathrm{TM}_{011}$ mode, and the inductive and capacitive effects cancel. Tuning was accomplished by the removal of $\sim 1 \mathrm{~mm}$ of material from a radius of 18 to 38 $\mathrm{mm}$ of the cathode. The perturbation to the magnetic field dominates and the position of the laser cathode surface relative to the cavity left unchanged. A summary of the old and new cavity parameters are given in table 1 .

Table 1: Cavity Parameters

\begin{tabular}{|l|l|l|}
\hline Parameter & Old Design & With rf seal \\
\hline Theoretical Q & 9596 & 13676 \\
\hline Q loaded (meas.) & 4700 & 7100 \\
\hline Coupling $(\beta)$ & 1.08 & 0.8 \\
\hline
\end{tabular}

A new plot of the field balance as a function of the frequency separation of the pi and zero modes[5] was created from Superfish calculations, and is shown in Figure 3 .

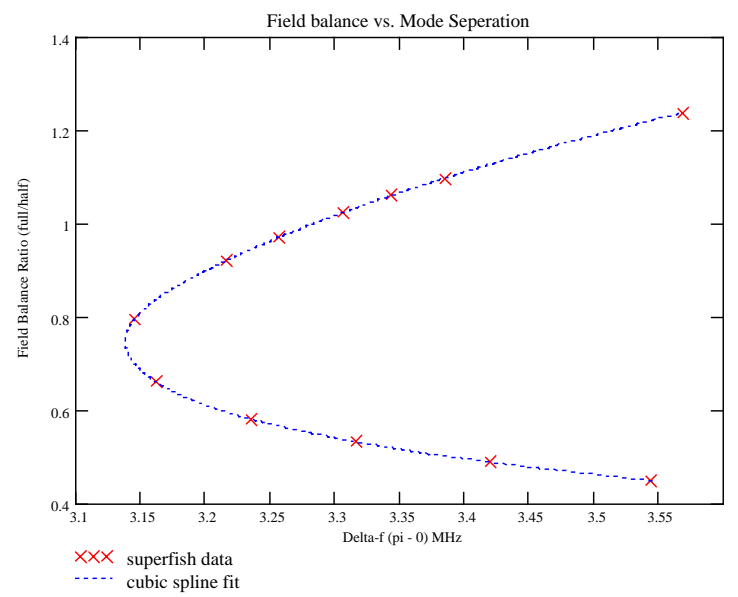

Figure 4: Field balance (full/half) vs. mode separation.

The rf coupling constant, $\mathrm{k}$, between cells is given by

$$
\omega_{m}^{2}=\omega_{o}^{2}\left\{1-k\left(1-\cos \left(\frac{m \pi}{N}\right)\right)\right\}
$$

where $\mathrm{m}$ is the $\mathrm{m}^{\text {th }}$ mode, $\mathrm{N}$ is the number of cells. For the new photocathode geometry this gives a coupling of $\mathrm{k}=0.00115$. This plot indicates a lower cell to cell coupling than in the original gun geometry.

\section{CAVITY TUNING}

The cavity was tuned by tightening the cathode plate bolts which adjusts the distance of the cathode with respect to the cavity body within the compliance range of the rf and vacuum seals. Ordinarily the tuning is such to achieve balanced fields, which for the above case is in the upper half of the field balance curve and a mode separation of $3.28 \mathrm{MHz}$. An additional constraint is that the compression of the spring seal should be between 10$30 \%$. Since the spring height is .051 inches, this corresponds to a range of .01 inches. The cavity as built differs from the model sufficiently that the cathode plate 
required several iterations on the cathode plate dimensions. During this process the tuning overshot the goal and ended up on the lower half of the curve, with a mode separation of $3.2 \mathrm{MHz}$ which corresponds to a field imbalance of 0.6. This has several implications which were quantified. First, since the field is tilted (higher) toward the half cell, the field is weaker in the full cell where the waveguide coupling iris is located. Also, the addition of the RF seal raised the $\mathrm{Q}$ of the pi mode. Both of these result in a weaker coupling $(\beta)$ to the waveguide feed from the klystron, and this was measured to be 0.83 , undercoupled.
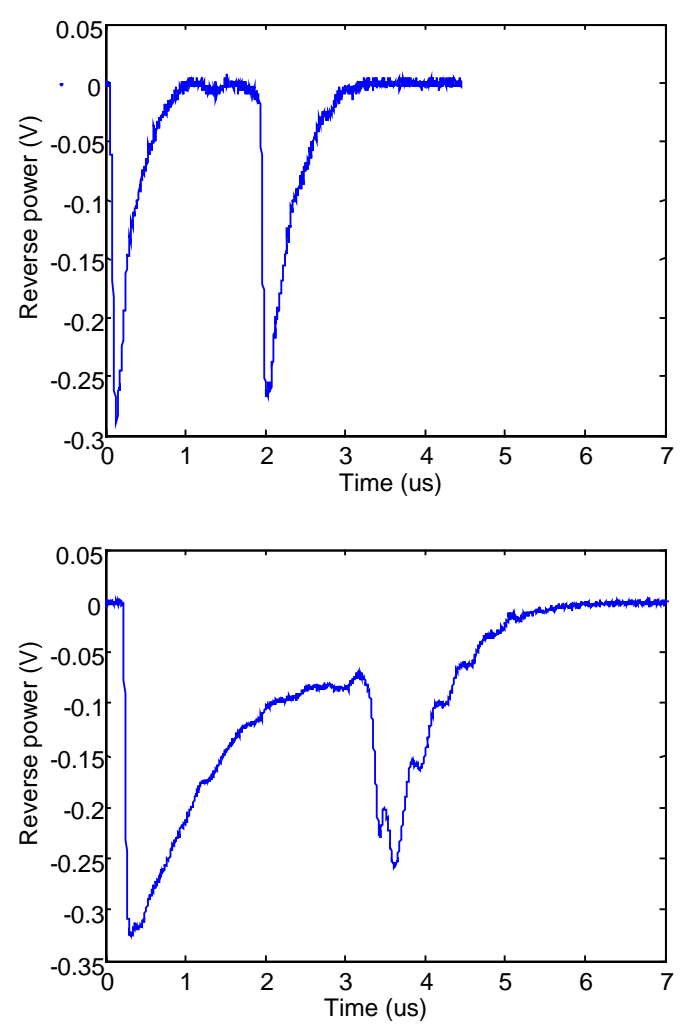

Figure 5. Reflected power as measured at the waveguide feed to the photocathode. Top trace: Original cathode, Bottom trace: New cathode with rf seal (Undercoupled).

The transmission efficiency from the klystron to the cavity is now only $90 \%$ of the $7.6 \mathrm{MW}$ available, or $6.8 \mathrm{MW}$. The loaded Q was measured to be 7100 . Hence the unloaded $\mathrm{Q}$ is

$$
Q_{o}=Q_{L}(1+\beta)=12780
$$

or $93 \%$ the theoretical value. This corresponds to a field on the cathode of $135 \mathrm{MV} / \mathrm{m}$, and $79 \mathrm{MV} / \mathrm{m}$ in the full cell.
The rf seal carries more than 2000 Amps/cm peak current, at $.003 \%$ duty factor. The successful high power test is a useful benchmark of the peak current capabilities of spring seals.

\section{HIGH POWER TESTING}

The new cathode was installed, the cavity tuned and tested at high power. One immediate improvement was that where it used to take 4-5 days to reach full power we spent less than one day with the new cathode. Although several sparks were monitored during the conditioning and subsequent week spent running with beam, when the cathode was disassembled and inspected there was no evidence of pitting on the cathode surface. A new cathode was constructed and has been installed and tuned for balanced field by adjusting the cathode position to obtain a $\pi-0$ mode separation of $3.28 \mathrm{MHz}$. for balanced fields for the 2001-2002 run.

\section{REFERENCES}

[1] L.H. Yu, L.DiMaro, A.Doyuran, W.S.Graves, E.D.Johnson, S. Krinsky, G. Rakowsky, T. Shaftan, B. Sheehy, J. Skaritka, J.H.Wu "The DUV-FEL Development Program" These proceedings

[2] W.S. Graves, L, DiMauro, D. Dowell, P.Emma, A.Doyuran,R.Heese,E.D.Johnson,S.Krinksy,J.Rose,J. Rothman,J.Rudati,J.Skaritka,T. Shaftan,B.Sheehy,L.H.Yu "Measured Propoerties of the DUVFEL High Brightness, Ultrashort Electron Beam" These proceedings

[3] W.S.Graves, D.Dowell, R.Heese,E.D.Johnson,J.Rose,T.Shaftan,B.Sheehy,L.H. $\mathrm{Yu}$ "DUVFEL Photoinjector Dynamics: Measurement and Simulation" These proceedings

[4] E.D.Johnson,L.DiMauro,A.Doyuran,K.Feng, W.S.Graves, R.Heese,S.Krinsky,C.Neuman,J.Rose,J.Rothman, J.Rudati, J.Skaritka, T.Shaftan, B.Sheehy, X.Wang, L.H.Yu "Commissioning Status of Source Development Laboratory At BNL" These proceedings. [5] D. T. Palmer, R.H. Miller, H. Winick, X.J. Wang, K. Batchelor, M. Woodle, I. Ben-Zvi, "Microwave Measurements of the BNL/SLAC/UCLA 1.6 Cell Photocathode RF Gun" Particle Accelerator Conf 1995

[6] K. Halbach, R.F. Holsinger, "SUPERFISH-A Computer Program for the Evaluation of RF Cavities with Cylindrical Symmetry”, Particle Accelerators 7 (4) (1976) 